\title{
AVALIAÇÃO DA QUALIDADE DE JABUTICABAS (CV. SABARÁ), SUBMETIDAS AO ARMAZENAMENTO REFRIGERADO E ACONDICIONADAS EM DIFERENTES EMBALAGENS
}

Quality assessment of jabuticabas (cv. sabará), submitted to refrigerated storage and conditioned in different packaging

Evaluación de la calidad de jabuticabas (cv. sabará), submetidas al almacenamiento refrigerado y acondicionadas en diferentes embalajes.

Cristiane Maria Ascari Morgado ${ }^{1}$, Bruna Ariel Dias Guariglia ${ }^{1}$, Rafaela Vieira Façanha ${ }^{2}$, Marcos José Trevisan ${ }^{3}$, Angelo Pedro Jacomino ${ }^{3}$, Gilmarcos de Carvalho Corrêa ${ }^{1}$, Luis Carlos Cunha Junior $^{*}$

${ }^{1}$ Universidade Federal de Goiás, Escola de Agronomia, Campus Samambaia, Goiânia, Goiás, Brasil.

${ }^{2}$ Universidade Estadual do Ceará, Centro de Ciências da Saúde, Cratéus, Ceará, Brasil.

${ }^{3}$ Universidade de São Saulo, Escola Superior de Agricultura “Luiz de Queiroz”, Piracicaba, São Paulo, Brasil.

*Correspondência: Universidade Federal de Goiás, Escola de Agronomia, Campus Samambaia, Av. Esperança, s/n, Goiânia, Goiás, Brasil.CEP:74.690-900.e-mail cunhajunior.l.c@gmail.com

\section{RESUMO}

A jabuticaba [Myrciaria jaboticaba (Vell) Berg] 'Sabara' é um fruto muito apreciado in natura, pelo seu sabor adocicado, porém é extremamente perecível. O objetivo deste trabalho foi avaliar embalagens para a conservação dos frutos sob refrigeração. Os frutos foram colhidos no ponto de consumo, lavados, higienizados, acondicionados nas embalagens PET com tampa Neoform N-90@; PVC 12 micras; PD- 900®; PEBD 15 micras; PP 10 micras; Controle (sem embalagem) e armazenados em câmara fria a $12^{\circ} \square 1^{\circ} \mathrm{C}$ e $85 \square 5 \%$ UR. As embalagens reduziram a perda de massa e mantiveram a firmeza das jabuticabas. O filme de PVC proporcionou melhor conservação de ácido ascórbico e luminosidade dos frutos. A atmosfera passiva no interior das embalagens PEBD e PP apresentaram as maiores concentrações de $\mathrm{CO} 2$ e menores de $\mathrm{O} 2$ do que as outras embalagens, afetando de maneira negativa a conservação das jabuticabas. A embalagem PVC de 12 micras, associada à refrigeração apresentou bons resultados para conservação das jabuticabas 'Sabará'.

Palavras-chave: Myrciaria jaboticaba (Vell) Berg, embalagem, refrigeração.

\section{ABSTRACT}

The jabuticaba [Myrciaria jaboticaba (Vell) Berg] 'Sabara' is a very popular fruit in natura, because its sweet taste, however it is extremely perishable. The aim of this study was to evaluate packages for storing fruit under refrigeration. The fruit was harvested at the point of consumption, washed, cleaned and stored in packages of PET with cover of Neoform N-90@; PVC 12 micron; PD-900®; PEBD 15 micron; PP 10 micron; Control (without package) and stored in chamber at $12^{\circ} \square 1^{\circ} \mathrm{C}$ e $85 \square 5 \%$ RH. The packages reduced the weight loss and contributed to maintenance the good appearance of jabuticabas. The PVC film showed better retention of ascorbic acid, appearance and brightness. The passive atmosphere inside the packages PEBD and PP showed the highest concentrations of $\mathrm{CO} 2$ and $\mathrm{O} 2$, negatively affecting the conservation of jabuticabas. The package PVC with 12 micron associated with cooling showed promising results for conservation of jabuticabas 'Sabará'.

Keywords: Myrciaria jaboticaba (Vell) Berg, packages, refrigeration. 


\section{RESUMEN}

"Sabara" es un fruto muy apreciado in natura, por su sabor dulzón, pero es extremadamente perecedero. El objetivo de este trabajo fue evaluar envases para la conservación de los frutos bajo refrigeración. Los frutos fueron recolectados en el punto de consumo, lavados, higienizados, acondicionados en los envases PET con tapa Neoform N-90 @); PVC 12 micras; PD-900®; PEBD 15 micras; PP 10 micras; Control (sin embalaje) y almacenados en cámara fría a $12^{\circ} \pm 1{ }^{\circ} \mathrm{C}$ y $85 \pm 5 \%$ UR. Los envases redujeron la pérdida de masa y mantuvieron la firmeza de las jabuticabas. La película de PVC proporcionó una mejor conservación de ácido ascórbico y luminosidad de los frutos. La atmósfera pasiva en el interior de los envases PEBD y PP presentaron las mayores concentraciones de $\mathrm{CO} 2$ y menores de $\mathrm{O} 2$ que los demás envases, afectando de manera negativa la conservación de las jabuticabas. El embalaje PVC de 12 micras, asociado a la refrigeración, presentó buenos resultados para la conservación de las jabuticabas 'Sabará'.

Descriptores: Myrciaria jaboticaba (Vell) Berg, embalaje, refrigeración.

\section{INTRODUÇÃO}

As jabuticabeiras pertencem à família Myrtaceae e são originárias da região centro sul do Brasil, podendo ser encontradas desde Minas Gerais até o Rio Grande do Sul (ASCHERI et al., 2006; SILVEIRA et al., 2006). As maiores produções concentram-se nos estados de São Paulo, Rio de Janeiro, Minas Gerais e Espírito Santo (SATO e CUNHA, 2007).

O potencial econômico de comercialização deste fruto é grande, devido às características sensoriais para consumo in natura ou usada na forma de sucos, geleias, licor e vinhos (WU et al., 2012). Possui casca fina, coloração roxa e adstringente que cobre uma polpa doce, branca e gelatinosa (TEIXEIRA et al., 2011); é rica em antocianinas e flavonoides (REYNERTSON et al., 2006). Esses compostos possuem propriedades biológicas bastante conhecidas como uma forte capacidade antioxidante e anti-inflamatória (Wu et al., 2012). Devido a esse alto potencial de aplicações industriais, a jabuticaba tem ganhado bastante interesse comercial.

Entretanto, ela é um fruto extremamente perecível e sua vida de prateleira é de apenas 2 a 3 dias, quando armazenada sob temperatura ambiente, o que dificulta sua comercialização (REYNERTSON et al., 2006). A redução na qualidade é associada à rápida alteração da aparência, decorrente da intensa perda de água, enrugamento da casca e perda de peso; e também à fermentação da polpa (BARROS et al., 1996). Assim, algumas tecnologias pós-colheita, como a refrigeração e o uso da atmosfera modificada são fundamentais para prolongar o período de comercialização de jabuticabas.

$\mathrm{O}$ uso de refrigeração é um dos meios mais eficazes para prolongar o tempo de comercialização com manutenção da qualidade, cuja função é retardar os processos metabólicos (CHITARRA e CHITARRA, 2005; KADER, 2002). Segundo Duarte et al. (1996), a melhor temperatura para o armazenamento de jabuticabas maduras foi $12^{\circ} \mathrm{C}$, após serem recobertas por ceras e/ou embaladas com filme plástico, o que permitiu um prolongamento da vida útil dos frutos por três semanas. Entretanto, a refrigeração isoladamente não é suficiente para estender a vida de prateleira dos frutos.

A associação da refrigeração com embalagens plásticas diminui o risco de contaminação, perda de massa e reduz o metabolismo, através da modificação passiva da atmosfera (MOSCA e VICENTINI, 2000; CHITARRA e CHITARRA, 2005). A atmosfera modificada baseia-se na combinação das características de permeabilidade do filme utilizado com a taxa de respiração e produção de etileno dos frutos. Havendo essa correta combinação, propicia-se uma atmosfera adequada dentro da embalagem para 
ocorrer consumo de $\mathrm{O}_{2}$ e produção de $\mathrm{CO}_{2}$ pela respiração (KADER, 2002).

Portanto, o objetivo deste trabalho foi avaliar o efeito de embalagens na vida-útil pós-colheita de jabuticabas 'Sabará', armazenadas a $12 \pm 1{ }^{\circ} \mathrm{C}$ e $85 \pm$ $5 \%$ UR.

\section{MATERIAIS E MÉTODOS}

Jabuticabas 'Sabará' [Myrciaria jaboticaba (Vell) Berg] foram colhidas no estádio de maturação comercial (completamente roxa), lavadas em água corrente e higienizadas com hipoclorito de sódio a 200 mg L-1 por 3 minutos. Após a higienização, um lote de jabuticabas foi acondicionado em bandejas de tereftalato de polietileno transparente, com tampa Neoform $\mathrm{N}-90^{\odot}$ (PET), outro lote foi acondicionado em contentores de poliestireno expandido e embalados com os filmes: policloreto de vinila de 12 micras da Goodyear $^{\circledR}$ (PVC); poliolefínico PD 900 da Cryovac ${ }^{\circledR}$; polietileno de baixa densidade de 15 micra (PEBD); polipropileno de 10 micra (PP); e um grupo permaneceu em bandejas sem embalagem (Controle). Todos as embalagens foram armazenadas a $12 \pm 1{ }^{\circ} \mathrm{C}$ e $85 \pm 5 \%$ UR, por até 6 dias. A unidade experimental foi uma embalagem com aproximadamente $200 \mathrm{~g}$ de jabuticabas, sendo utilizadas três repetições por tratamento para cada dia de análise.

A cada dois dias, os frutos foram avaliados quanto a perda acumulada de massa fresca, com o auxílio de uma balança eletrônica, Tecnal modelo BG 2000, e os resultados foram expressos em porcentagem. Avaliou-se a composição atmosférica inicialmente e após 12, 24, 48, 96 e 144 horas usandose um analisador de gases (Dansensor, modelo Checkmate 9001, Dinamarca).

A evolução da coloração foi avaliada com colorímetro MINOLTA CR 300 (MINOLTA CORP, 1994), na qual foram determinados os valores de luminosidade, sendo realizada na região equatorial em 10 jabuticabas por repetição (embalagem), perfazendo um total de 30 repetições por tratamento. Nestes frutos foi determinada também a pressão de turgescência através da técnica de aplanação, descrita por Calbo e Nery (1995).

Os teores de sólidos solúveis (SS) foram determinados em refratômetro digital Atago, PAL-1 (AOAC,1997) e de acidez titulável (AT) com base no método da AOAC (1997); e calculou-se a relação SS/AT com base no método da AOAC (1997). O teor de ácido ascórbico (AA) foi determinado por titulometria, na qual 5 gramas da polpa foram diluídas com $25 \mathrm{~mL}$ de ácido oxálico a $1 \%$ refrigerado $\left(5^{\circ} \mathrm{C}\right)$, e posteriormente titulados com reagente de 2,6 diclorofenolindofenol sódio (CARVALHO et al., 1990). O experimento foi conduzido em esquema fatorial $(6 \times 4)$ composto por dois fatores: embalagem (Controle, PET, PD-900, PVC, PEBD e PP) e dias de armazenamento ( $0 ; 2 ; 4$ e 6 dias).

\section{RESULTADOS E DISCUSSÃO}

O estádio de maturação no qual o fruto é colhido tem fundamental importância no tempo de comercialização (CHITARRA e CHITARRA, 2005). A jabuticaba é uma fruta climatérica, portanto deve ser colhida no ponto de maturidade fisiológica que, neste caso, coincide com o seu ponto de consumo, no entanto devido as suas características, como alto teor de água e casca fina essa fruta tem uma vida útil muito curta.

A maior perda acumulada de massa fresca foi de $4 \%$ nos frutos do tratamento Controle (Figura 1), os quais apresentaram uma maior inclinação da reta $(\mathrm{y}=$ $0,668 x+0,071)$ ao longo do armazenamento. Este comportamento matemático é proporcional a intensidade da perda de massa fresca. Já os frutos que receberam algum tipo de embalagem mantiveram baixos índices de perda de massa fresca, visto que as embalagens são barreiras seletivas à troca de vapor de água (FABER, 1991), o que explica a menor perda de 
massa fresca das jabuticabas embaladas, em relação aos frutos do controle.

Comparando as embalagens, observou-se que elas apresentaram comportamento semelhante e foram eficazes na diminuição de perda de massa. As embalagens PD-900, PVC 12 e PEDB apresentaram a mesma equação da reta para esta variável, seguida da embalagem PP e PET. Machado et al. (2007) observaram que o saco plástico de 15 micras reduziu significativamente a perda de massa fresca em jabuticaba quando comparada com frutos sem embalagem, o que foi semelhante ao encontrado neste trabalho. Agostini et al. (2009) relataram que tratamentos com embalagem de polietileno e PVC foram os mais eficientes na manutenção da umidade no interior da embalagem, acarretando com isso uma menor perda de massa. Sanches et al. (2015) observaram maior perda de massa fresca nos frutos que não receberam aplicação de ácido salicílico (Controle), o que foi semelhante ao encontrado nesse trabalho.

Figura 1. Perda acumulada de massa fresca (\%) em jabuticabas 'Sabará' acondicionadas em diferentes embalagens e armazenadas sob refrigeração $\left(12 \pm 1^{\circ} \mathrm{C}\right.$ e $85 \pm 5 \%$ UR).

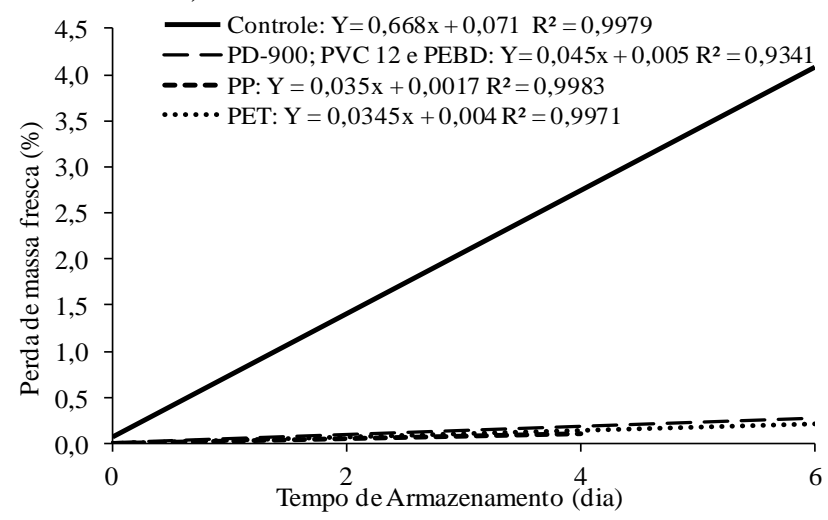

A perda de massa fresca reflete na turgidez dos frutos, podendo ser relacionada com a evolução da firmeza obtida pelo método da aplanação (CALBO e NERY, 1995). As jabuticabas do tratamento controle apresentaram a maior redução da massa fresca (Figura 1) e da firmeza (Figura 2). Essa redução na firmeza durante o período de armazenamento pode ser explicada visto que a etapa final de desenvolvimento de jabuticabas é marcada pela diminuição acentuada nos teores de pectina, como consequência do processo de amadurecimento (MAGALHÃES et al., 1996). Por outro lado, os frutos embalados apresentaram menor redução na firmeza, o que pode estar relacionado com a elevada turgidez de frutos.

A firmeza dos frutos é um importante atributo de qualidade, visto que frutos com valores de firmeza mais elevados são mais resistentes às injúrias mecânicas durante o transporte e a comercialização (BECKER et al., 2015).

Mota et al. (2002), utilizando a mesma metodologia para determinar a firmeza em jabuticabas da cv. Sabará, também observaram redução da turgidez ao longo do armazenamento. As embalagens PD 900, PVC e PEDB e PP proporcionaram aos frutos condições semelhantes em relação à redução de massa (Figura 1) e firmeza (Figura 2).

Figura 2. Firmeza $\left(\mathrm{Kgf} / \mathrm{cm}^{2}\right)$ de jabuticabas 'Sabará' acondicionadas em diferentes embalagens e armazenadas sob refrigeração $\left(12 \pm 1^{\circ} \mathrm{C}\right.$ e $85 \pm 5 \%$ UR).

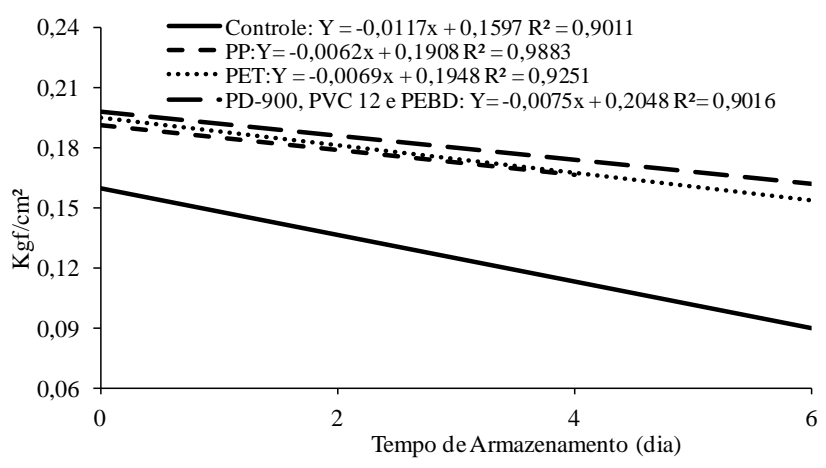

Em relação à seletividade aos gases $\mathrm{O}_{2}$ e $\mathrm{CO}_{2}$, os filmes PEBD 15 micras e PP 10 micras proporcionaram baixas concentrações de $\mathrm{O}_{2}$ e altos teores de $\mathrm{CO}_{2}$ no interior da embalagem. A composição gasosa na embalagem PEDB 15 micras foi de $11 \% \quad \mathrm{CO}_{2}$ e $5,2 \% \quad \mathrm{O}_{2}$ com 144 horas de armazenamento. $\mathrm{Na}$ embalagem $\mathrm{PP}$ estas concentrações foram de $16,6 \% \mathrm{CO}_{2} \mathrm{e}$ 6,4\% $\mathrm{O}_{2}$ (Figura 3). Essa forte mudança atmosférica dos filmes PP e 
PEBD influenciou negativamente o metabolismo dos frutos, os quais apresentaram odor alcoólico no quarto e sexto dia, respectivamente. Esse odor alcoólico provavelmente está relacionado ao metabolismo anaeróbico que ocorreu no interior das embalagens, devido à baixa concentração de oxigênio e/ou altas concentrações de $\mathrm{CO}_{2}$.

As demais embalagens (PET, PVC e PD-900) modificaram moderadamente os teores de $\mathrm{O}_{2}$ e de $\mathrm{CO}_{2}$ dentro da embalagem com benefícios para conservação, pois os teores de $\mathrm{O}_{2}$ não favoreceram condições de anaerobiose.

Figura 3. Evolução da pressão parcial de oxigênio (A) e dióxido de carbono (B) em jabuticabas 'Sabará' acondicionadas em diferentes embalagens e armazenadas sob refrigeração $\left(12 \pm 1^{\circ} \mathrm{C}\right.$ e $85 \pm 5 \%$ UR $)$.
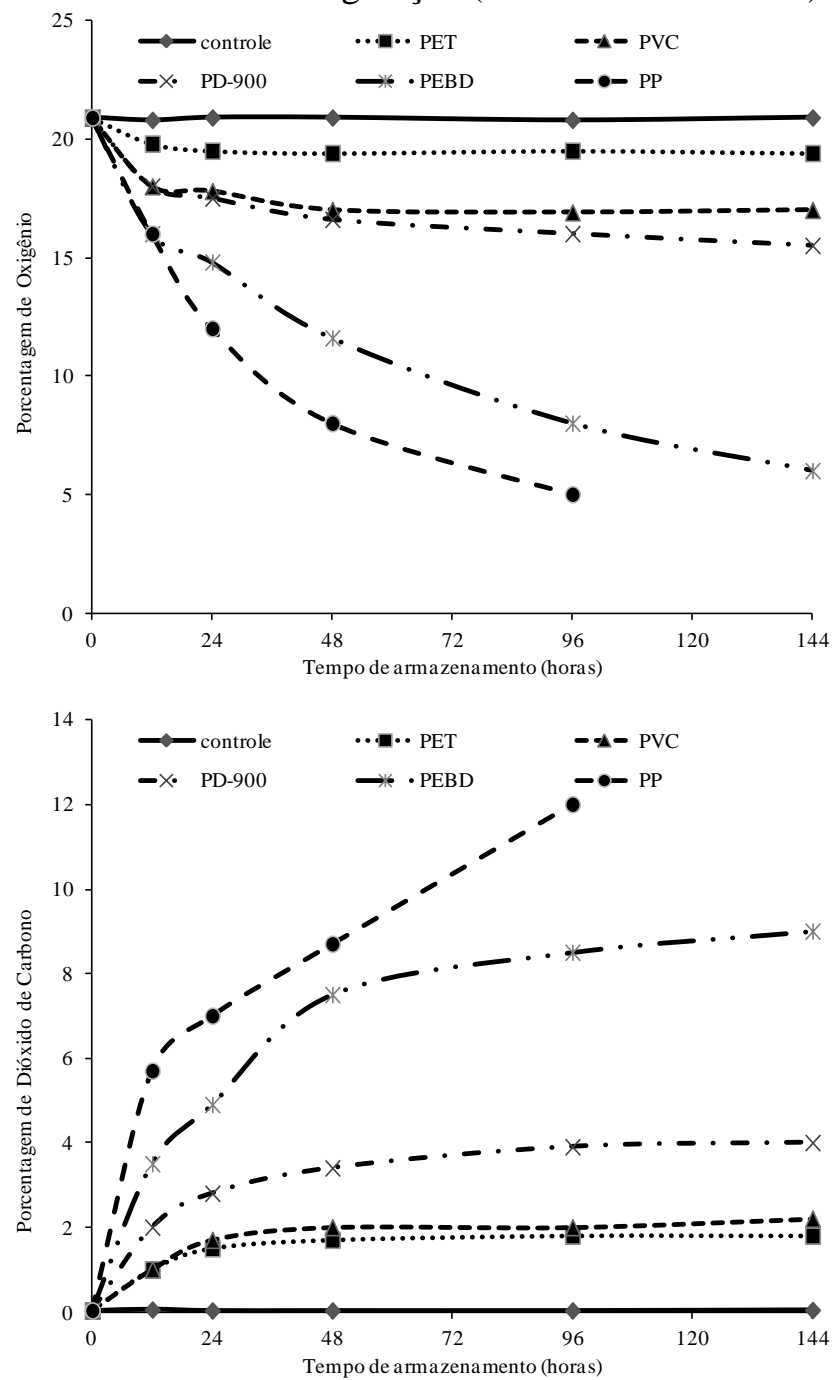

Quanto aos valores de luminosidade observouse redução em todos os tratamentos testados (Figura
4). Os frutos acondicionados em PVC 12 obtiveram a menor perda de brilho. A maior redução do brilho ocorreu em jabuticabas acondicionadas em embalagem PP, o que pode ser efeito da grande mudança da atmosfera dos frutos que provocou mudanças do $\mathrm{pH}$, e que pode ter alterado a coloração da casca da jabuticaba (REYNERTSON et al., 2006). De acordo com Março et al. (2008), o teor de antocianinas pode mudar conforme o $\mathrm{pH}$ do meio, ocasionando assim mudanças na coloração do fruto. Os frutos controle apresentaram valores de perda de brilho intermediários entres os valores das embalagens PP e PVC 12. De modo contrário, Teixeira et al. (2011) observaram que jabuticabas submetidas a tratamentos com 1 e $5 \mathrm{Ka} \quad \mathrm{O}_{2}$ tornaram-se mais brilhantes no fim do armazenamento ( 8 dias) a $12^{\circ} \mathrm{C}$.

Figura 4. Luminosidade da casca de jabuticabas 'Sabará' acondicionadas em diferentes embalagens e armazenadas sob refrigeração $\left(12 \pm 1^{\circ} \mathrm{C}\right.$ e $85 \pm 5 \%$ UR).

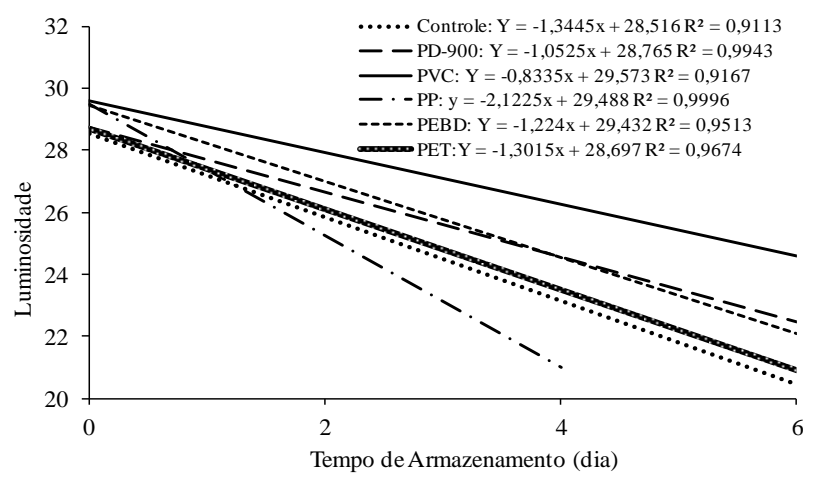

Os teores de sólidos solúveis não foram influenciados pelos fatores estudados (embalagens e dias de armazenamento), com os valores médios variando de 13 a $15^{\circ}$ Brix (dados não mostrados). Os teores encontrados neste trabalho estão dentro da faixa de 11,5 a $20^{\circ}$ Brix e corroboram com diversos autores em estudos com jabuticabas 'Sabará' (OLIVEIRA et al., 2003; BRUNINI et al., 2004; AGOSTINI et al., 2009; VIEITES et al., 2011). A variação de $2^{\circ}$ Brix encontrada durante o armazenamento é característico de frutas não climatéricas, que são colhidas na 
maturidade fisiológica e apresentam pouca variação no armazenamento (CHITARRA e CHITARRA, 2005).

Os teores de acidez titulável dos frutos (dados não apresentados) não diferiram estatisticamente entre as embalagens, entretanto ocorreu aumento ao longo do armazenamento que variou de 0,61 a $0,79 \mathrm{~g}$ de ácido cítrico por $100 \mathrm{~g}$ de polpa. Este teor de acidez titulável é superior ao relatado por Machado et al. (2007), que encontraram uma faixa de 0,28 a $0,51 \mathrm{~g}$ de ácido cítrico por $100 \mathrm{~g}$.

A relação entre os teores de sólidos solúveis e acidez titulável ('Ratio') é considerada como um critério de avaliação do sabor em vários frutos, além de ser indicativa do nível de amadurecimento e do grau de doçura (CHITARRA e CHITARRA, 2005). Os valores iniciais do 'ratio' foram de 25,2 e no final do armazenamento de 18,1 (Figura 5A), valores estes intermediários aos encontrados por Brunini et al. (2004) e Machado et al. (2007), com valores médios para o ratio de 15 e 42 , respectivamente. A redução do ratio sugere uma leve redução na qualidade organoléptica dos frutos, sendo o principal contribuinte o aumento dos teores de ácidos orgânicos.

Figura 5. Valores de ratio (A) e teores de ácido ascórbico (B) (mg de ácido ascórbico por $100 \mathrm{~g}$ de polpa) em jabuticabas 'Sabará' acondicionadas em diferentes embalagens e armazenadas sob refrigeração $\left(12 \pm 1{ }^{\circ} \mathrm{C}\right.$ e $85 \pm 5 \%$ UR).

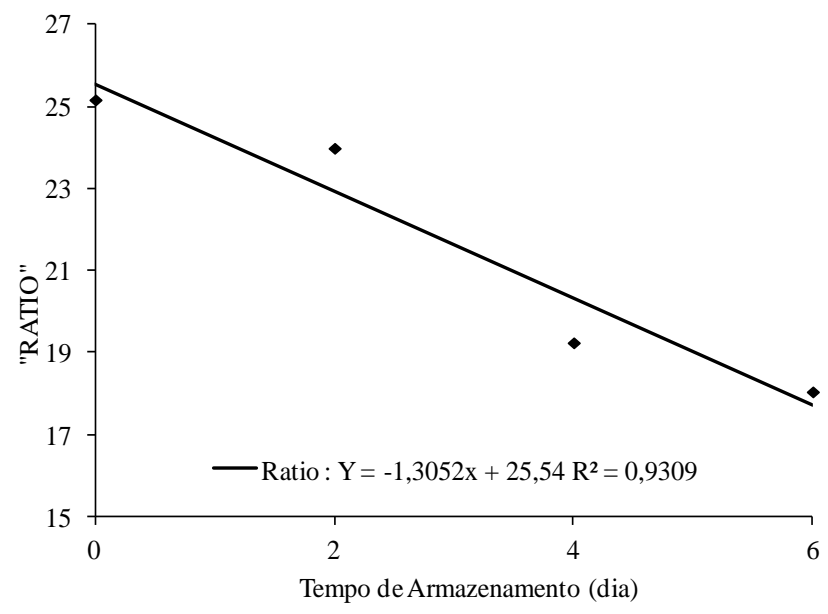

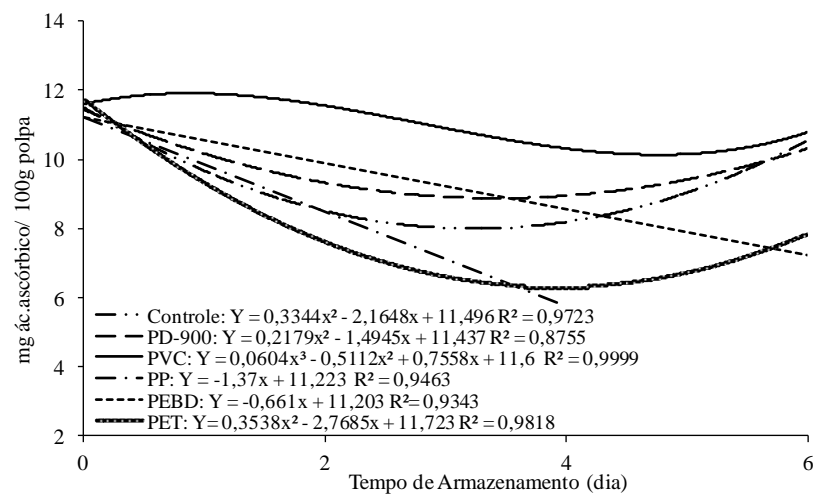

As frutas constituem uma fonte rica de vitaminas e minerais, na qual se destaca a vitamina $\mathrm{C}$, sendo um importante fator na nutrição humana (KADER, 2002). O ácido ascórbico é o principal componente da vitamina $\mathrm{C}$, considerada como um excelente antioxidante. Este composto é considerado um parâmetro de qualidade pós-colheita no qual possui atuação nas reações redox na cadeia de transporte de elétrons e atua regenerando diferentes substâncias na célula (CHITARRA e CHITARRA, 2005).

Os frutos acondicionados nas embalagens PP e PEBD apresentaram decréscimos lineares de ácido ascórbico (Figura 5B), podendo ser uma resposta ao estresse causado pelo baixo teor de $\mathrm{O}_{2}$ e alto de $\mathrm{CO}_{2}$, o que ocasionou danos na cadeia de transporte de elétrons, e por sua vez, maior atividade deste antioxidante na reparação dos processos metabólicos envolvidos. As jabuticabas acondicionadas em embalagem PVC apresentaram a melhor manutenção do teor ácido ascórbico em relação aos demais tratamentos. Agostini et al. (2009), também observaram redução no teor de ácido ascórbico em jabuticabas cv Paulista refrigeradas ou não e com ou sem recobrimentos. Brunini et al. (2004) também constataram redução no teor de ácido ascórbico em jabuticabas da cultivar 'Sabará' ao final do período de armazenamento.

\section{CONCLUSÃO}

As embalagens proporcionaram menor perda de massa fresca em das jabuticabas 'Sabará', com 
exceção da embalagem PP. O filme policloreto de vinila de 12 micras (PVC) apresentou bons resultados na conservação das jabuticabas, possibilitando manter as características organolépticas e de qualidade do produto durante 6 dias de armazenamento refrigerado $\left(12 \pm 1^{\circ} \mathrm{C}\right.$ e $\left.85 \pm 5 \% \mathrm{UR}\right)$.

Todos os autores declararam não haver qualquer potencial conflito de interesses referente a este artigo.

\section{REFERÊNCIAS}

AGOSTINI, J. S.; CANDIDO, A. C. S.; TEODÓSIO, T. K. C.; RODRIGUES, J. N.; GARCETE, G. J.; SCALON, S. P. Q. Atmosfera modificada e condições de armazenamento nas características físico-químicas de jabuticabas da cultivar 'paulista'. Ciência Rural, v.39, n.9, p.2601-2608, 2009.

AOAC. Official methods of analysis of the Association of Analytical Chemists. 13ed.Washington, 1997. 1018p.

ASCHERI, D.P.R.; ASCHERI, J.L.R., CARVALHO, C.W.P. Caracterização da farinha de bagaço de jabuticaba e propriedades funcionais dos extrusados. Ciência e Tecnologia de Alimentos. v.26, n.4, p.897905, 2006.

BECKER, F.S.; VILAS BOAS, A.C.; SALES, A.; TAVARES, L.S.; SIQUEIRA, H.H.; VILAS BOAS, E.V.B. Characterization of 'Sabará' Jabuticabas at different maturation stages. Acta Scientiarum, v.37, n.4, p.457-462, 2015.

BARROS, R.S.; FINGER, F.L.; MAGALHÃES, M.M. Changes in non-structural carbohydrates in developing fruit of Myrciaria jaboticaba. Scientia Horticulturae, Amsterdam, v.66, p. 209-215, 1996. BRUNINI, M.A.; OLIVEIRA, A.L. de; SALANDINI, C.A.R.; BAZZO, F.R. Influência de embalagens e temperatura no armazenamento de jabuticabas (Myrciaria jabuticaba (Vell) Berg) cv 'Sabará. Ciência e Tecnologia de Alimentos, v.24, n.3, p.378383, 2004.

CALBO, A.G.; NERY, A.A. Medição de firmeza em hortaliças pela técnica de aplanação. Horticultura Brasileira, v.13, n.1, 1995.

CARVALHO, C.R.L.; Mantovani, D.M.B.; Carvalho, P.R.N.; Moraes, R.M.M. Análises químicas de alimentos. Campinas: ITAL, 1990. 121 p.
CHITARRA, M.I.F.; CHITARRA, A.B. Pós-colheita de frutos e hortaliças: fisiologia e manuseio. Lavras: ESAL/FAEPE, 2005, 2 ed., 320p.

DUARTE, O.; LUDDERS, P.; HUETE, M. Extending storage life of jaboticaba (Myrciaria cauliflora Berg) fruits. 14 Congresso Brasileiro de Fruticultura, 1996, Curitiba, PR, Resumos... Curitiba-PR: SBF, p.556, 1996.

FARBER, J.M. Microbiological aspects of modified atmosphere packing technology- a review. Journal of Food Protection, v.54, n.1, p.58-70, 1991.

KADER, A.A. (Ed.) Postharvest technology of horticultural crops. 3 ed. Davis: University of California, 2002. 535p.

MACHADO, I.P.; COUTINHO, E.F.; CAETANO, E.R. Embalagens plásticas e refrigeração na conservação pós-colheita de jabuticabas. Revista Brasileira de Fruticultura, v.29, n.1, p.166-168, 2007.

MAGALHÃES, M.M.; BARROS, R.S., FINGER, F.L. Changes in structural carbohydrates in developing fruit of Myrciaria jaboticaba. Scentia Horticulturae, v.66, p.17-22, 1996.

MARÇO, P. H.; POPPI, R.J.; SCARMINIO, I. S. Procedimentos analíticos para identificação de antocianinas presentes em extratos naturais. Quimica Nova, v. 31, n.5, p.1218-1223, 2008.

MINOLTA CORP. Precise color communication: color control from feeling to instrumentation. Japão, 1994. $49 \mathrm{p}$.

MOSCA, J.L., VICENTINI, N.M. Embalagens utilizadas na conservação pós-colheita de frutas e hortaliças in natura. Jaboticabal: Funep, 2000. 21p.

MOTA, W.F.; SALOMÃO, L.C.C.; PEREIRA, M.C.T.; CECON, P.R. influência do tratamento póscolheita com cálcio na conservação de jabuticabas. Revista Brasileira de Fruticultura, v.24, n.1, p.4952, 2002.

OLIVEIRA, A.L.; BRUNINI, M.A.; SALANDINI, C.A.R.; BAZZO, F.R. Caracterização tecnológica de jabuticabas 'Sabará' provenientes de diferentes regiões de cultivo. Revista Brasileira de Fruticultura, v.25, n.3, p.397-400, 2003.

REYNERTSON, K.A., WALLACE, A.M., ADACHI, S., GIL, R.R., YANG, H., BASILE, M.J, D'ARMIENTO, J., WEINSTEIN, I.B., KENNELLY, E.J. Bioactivede psides andanthocyanins from 
jaboticaba (Myrciaria cauliflora). Journal of Natural Products, v.69, p.1228-1230, 2006.

SATO, A.C.K.; CUNHA, R. L. Influência da temperatura no comportamento reológico da polpa de jabuticaba. Ciência e Tecnologia de Alimentos, v.27, n.4, p.890-896, 2007.

SANCHES, A.G.; SILVA, M.B.; MOREIRA, E.G.S.; CORDEIRO, C.M.A. Qualidade e resistência póscolheita de jabuticabas tratadas com ácido salicílico. Acta Iguazu, v.4, n.4, p.28-40, 2015.

SILVEIRA, F.T.; ORTOLANI, F.A.; MATAQUEIRO, M.F.; MORO, J.R. Caracterização citogenética em duas espécies do gênero Myrciaria. Revista de Biologia e Ciências da Terra, v.6, p.327333, 2006.

TEIXEIRA， G.H.A.; DURIGAN， J.F.; SANTOS, L.O.; HOJO, E.T.D.; CUNHA JUNIOR, L.C. Changes in the quality of jaboticaba fruit (Myriciaria jaboticaba (Vell) Berg. cv. Sabará) stored under diferent oxygen concentrations. Journal of the Science of Food and Agriculture, v.91, n.15, p.28442849, 2011.

VIEITES, R.L.; DAIUTO, E.R.; MORAES, M.R.; NEVES. L.C.; CARVALHO, L.R. Caracterização físico-química, bioquímica e funcional da jabuticaba armazenada sob diferentes temperaturas. Revista Brasileira de Fruticultura. v.33, n.2, p 362-375, 2011.

WU, S.B.; DASTMALCHI, K.; LONG, C.; KENNELLY, E. J. Metabolite profiling of jaboticaba (Myrciaria cauliflora) and other dark-colored fruit juices. Journal of Agriculture and Food Chemistry, v.60, n.30, p.7513-7525, 2012. 